\title{
Component Based Representation Using Probabilistic Neural Network Classifier
}

\author{
Anjali G \\ (Computer science,Caarmel Engineering College,India
}

\begin{abstract}
The law enforcement agency make use of face recognition system for the identification of criminals. In some cases, the photograph of criminals is not available. In this situation, sketch is drawn by the information provided by the eyewitness. In the early stages sketches are drawn by the artist by the information provided by the eyewitness. Sometimes the witness does not recall the exact apperance of the suspect. In that case,sketch drawn is not helpfull for investigation. In this networked world, the sketch construction with the help of computer generated software arises. The computer generated composite sketch is constructed by selecting the facial components provided in the system database. The composite sketch construction gives more faster results for the law enforcement agency. The component based approach for face recognition using probabilistic neural network classifier gives more accurate result in photo to sketch matching. The proposed approach performs facial decomposition, some transformations and feature extraction for photo to composite matching. The use of classifier is helpfull for comparing the features of face images and gives the degree of match to make a decision. The advantage of using Probabilisitic Neural Network (PNN) classifier is that it requires only short training time.
\end{abstract}

Keywords: Composite based approach, composite sketch , PNN

\section{Introduction}

Law enforcement agency make use of sketches for identifying criminals. Depending on the context of an application face recognition system can be either a one to one or a one to many system. The one to one comparison is to determine whether the person presenting to the system is the person who claims to be. A one to many search recognises a person by checking the entire template database for a match. Face recognition is a visual pattern recognition problem. A face recognition system with the input of an arbitrary image will search in database to output peoples identication in the input image. Facial sketches are commonly used in law enforcement to assist in identifying suspects involved in a crime when no facial image of the suspect is available at the crime scene. After the sketch is created, enforcement agency place the sketch to public in hope that anyone will recognize the individual and give any information that help for investigation. Hand drawn sketches are drawn by artist either by seeing the photograph or by the person itself. Forensic sketch are drawn by artist provided by the information given by the witness. Sometimes the witness doesn't recall the exact appearance of victim this will lead to mismatch between understanding and depiction. Facial sketches are particularly valuable when eyewitnesse's descriptions are available[1]. This process is insufficient and does not leverage all available resources, the extensive mugshot databases maintained by law enforcement agencies. Hand drawn sketches capture the most distinctive characteristics of facial components. Hand drawn sketches is more time consuming. Law enforcement agency maintain a database of suspect. The purpose of maintaining the database is to allow law enforcement to have a photographic record of the arrested individual to allow for identication by victims and investigator. Forensic sketch artists typically require a few years of training to become proefficient in drawing sketches[2].

In this networked world, law enforcement agency make use of computer generated sketches. Composite sketches are facial sketches created using software kits which allow an operator to select various facial components. Composite sketches have become a popular and more affordable alternative to forensic sketches. Composite sketches are created by the operator while viewing the photo or by the description provided by eyewitness. The composite sketch is constructed by selecting the facial components provided in the database.

Most of the software kits provide a predefined set of facial components. In component based approach,the method is to classify local facial components. Component based representation for composite sketches to face photos provide a common representation maintaining inter person discriminability. Component based approach compares local facial primitives individually. The component based face recognition system was compared to a whole face recognition system. The task of face recognition was simplified by choosing facial components instead of the whole face.

A face recognition system generally consists of four modules detection,alignment, feature extraction, and matching. The localization and normalization are preprocessing steps. After that facial feature extraction 
and matching is performed. The component based system will either make a match and subsequently identify the person or it will fail to make a match. The use of classifier in face recognition system is to compare the features of a face image with those of images in database and report the degree of match in terms of some match or similarity measure. In component based system uses classifier to handle variations in pose and scale of photo to sketch matching.

While using Support Vector Machine (SVM) classifier the face is represented as a combination of individual facial components and train seperate general SVM classi_ers for di_erent components. A simple linear SVM classifer can be used to determine if the combination of position and size of found components represent a face. An SVM was trained for each subject in the database to separate from all the other subjects. To determine the identity of a person at runtime, compare the normalized outputs of the SVM classifiers. The main disadvantage of using SVM classifier is the choice of kernel function.

Neural Network are widely used in face recognition system. Artifical neural network can predict not only the seen data but also the unseen data. It work well for linearly and non linearly seperable datasets. Neural network classifier may be supervised or unsupervised. Instead of using SVM classifier, the proposed methods use proababilistic neural network(PNN) classifier. The PNN can map any input pattern to a number of classifications. The main advantage is that the fast training process and it is instantaneous.

\section{Related Works}

The previous work focus on hand drawn sketches, forensic sketches and composite sketches. In the early stages, law enforcement agency make use of hand drawn sketches for criminal investigation. The sketches are drawn by the artist either by seeing himself or by seeing the photograph. The sketch are displayed to the public through medias in hope that any clue from person will help the investigation. The artist requires more time for drawing the sketch. The viewed sketches perform transformation that convert sketch to photos. This method uses eigen transformation, Principal Component Analysis(PCA).

Forensic sketches are drawn by a forensic artist using only on a verbal description. During the drawing of a forensic sketch, the witness usually cannot exactly recall the facial appearance of a suspect. Some disparity exists between the understanding and depiction of facial features between an artist and the eyewitness. Therefore, the additional challenges are posed when matching forensic sketches against face photographs. To handle the difficulties, develop a local feature based discriminant analysis. Forensic sketch artists typically require a few years of training to become proefficient in drawing sketches, only a few hours of training are required before a police officer becomes proefficient in using composite sketch software[3].

Composite sketches created using software kits allow an operator to select each facial components individually. Composite software kit allows to choose different facial components based on the information provided by the victims description. The objective is to improve the efficiency of the suspect identification process. Now a days law enforcement agency make use of computer generated sketch. This type of sketch generation gives faster results.

\section{3. Proposed System}
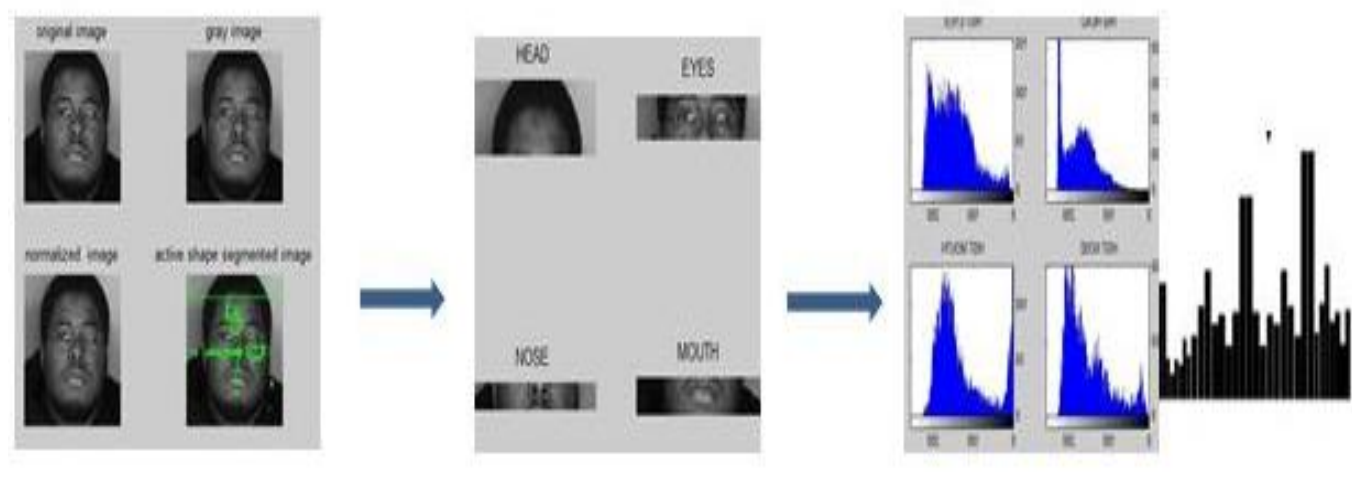

Fig 3.1 Architecture of the proposed system

Face recognition system is of manual or automated system. Composite sketch constructed by software kits uses component based representation for matching sketches to face photos. Component based representation first detect a set of facial landmarks by means of an active shape model. Then a per component feature extraction and similarity score of each facial component is calculated. A multiscale local binary pattern is used for extracting feature of each facial component. The component based face recognition system was compared to 
a whole face recognition system; both systems were trained and tested on the same images. The component based methoduses linear dicsriminant analysis to improve the recognition accuracy. Block based feature extraction is used to spatially encode the structural information of each component.

The input is a sketch constructed by a witness. The output is the matching score for the suspect database. A sketch matching algorithm using local and global features followed by relevance feedback from the users was proposed for recognizing composite sketches created and global feature comparison as distance measurements between faces. The local features computes facial primitives individually while the global features computes the geometric differences between feature points. Component based representation uses a grey scale image as input to the system.

The proposed system consists of four steps

1,Face Normalization

2.Component Localization

3.Feature Extraction

4.Similairity Calculation

3.1 Face Normalization

Convert all color images into greyscale because HSV model requires high computational cost and high processing . Certain geometric transformation like rotation and scaling is applied face images to handle variations in pose and scale. The transformation is applied to both face photos and composite sketches. Transformation is applied based on eye centers . While applying geometric transformation speed optimization is a problem for large set of images.

\subsection{Component localization}

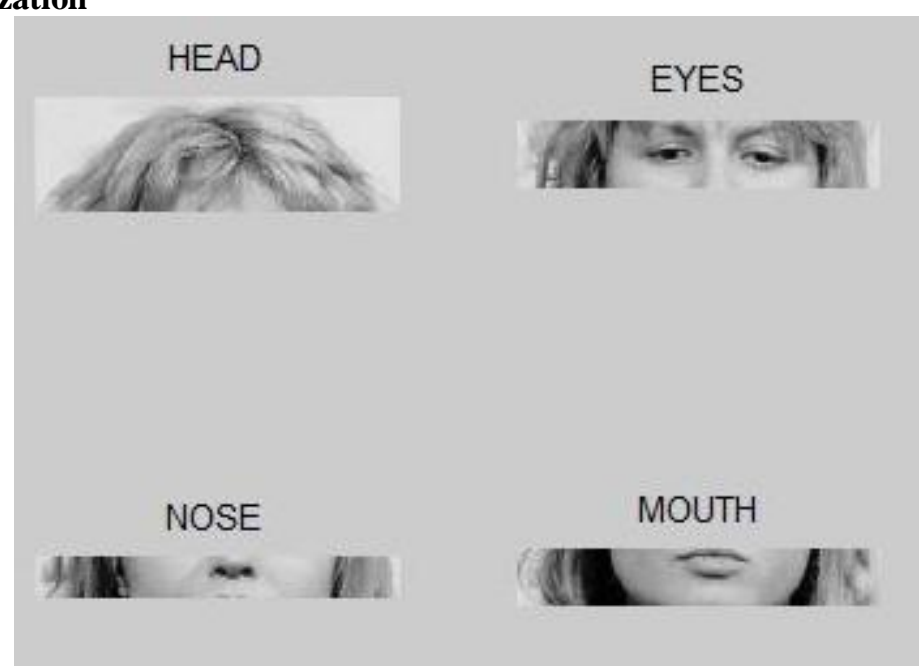

Fig 3.2.1 Facial Decomposition

This step detects a set of facial landmarks using an active shape model. The facial landmarks form a shape. The face is divided into different facial components. Normally images stored in the mugshot gallery are taken in frontal pose. ASM attempt to find the best match position between model and data in the new image. Active Shape Models are aimed at automatically locating landmark points that define the shape of any object in an image. When modeling faces, the landmark consist of points that lie along the shape boundaries of facial features such as the eyes, lips,nose, mouth and eyebrows as shown in fig 3.2.1. The training stage of an ASM involves thebuilding of a facial model from a training set containing images with landmarks. To handle variation in pose and scale in photo matching, a probabilistic neural network classifier is used. The PNN works on the basis of back propagation algorithm which help us to reduce the minimize the square error function. The neural network classifier is more accurate than all other classifier. This classifier work on the basis of supervised learning approach..

The network consists of an input layer, radial basis layer and competitive layer. Competitive layer makes a classification based on the radial basis unit with the largest output[4]. The first layer calculates a radial basis function, also called as kernel function, for distances between the input vector and each of the kernel vectors. The radial distance is given as argument to the function. The weight is calculated as a function of its the radial basis function. The function of hidden layer is unseen to the user. The weight is calculated as a function of its radial distance. The second layer computes $\mathrm{K}$ sums of the radial basis kernel outputs and basis kernel outputs and finds the largest sum. The class corresponding to the largest sum has the maximum probability of being 
correct and is given as the output of the network. A probabilistic neural network classi_er is used to handle large pose variations in photo matching. The advantage of using PNN classifier is that training time required is short and instantenous. Images in the mugshot gallery are taken in frontal pose. The pose and scale variations can be eliminated by the localization step,

3.3 Component Feature Extraction

The intensity variation in photos is due to shading of faces. The sketch and photos lack shading information. To avoid this a Local Binary Pattern(LBP) is used. The face area is first divided into small regions from which LBP histograms are extracted and concatenated into a single, spatially enhanced feature histogram for representing the face image. The original LBP operator is a powerful means of texture description. The operator labels the pixels of an image by thresholding the neighborhood of each pixel with the center value and considering the result as a binary number. Then the histogram of the labels can be used as a texture descriptor. A face image is first divided into several blocks and then extract local binary pattern and construct a global feature histogram and find the matching score. The histogram that represent both the statistics of the facial micro patterns and their spatial locations. Face image is divided into rectangular regions each of same size. The other regions are different sizes and shapes can be used. One of the drawback is in the length of feature vector used for face representation. The recognition is performed using a nearest neighbour classifier in the computed feature space.

LBP is insufficient at single scale to capture the features of all facial components, so a multiscale local binary pattern is introduced. MLBP help to capture all features in different facial components. To reduce the computational cost of feature extraction hair component can be scale down[5]. For all other components, increase the size of components then only apply block based feature extraction. For obtaining the spatial information decompose each facial component into overlapped blocks.

3.4 Similarity Calculation

Similarity of each facial component can be calculated based on normalized histogram intersection. Similarities of each block can be calculated. The total component similarity can be obtained by summation of similarities of all the blocks. For finding the matching score between the sketch and face photo sum up all the component similarities. Score normalization are applied because score obtained may not be in numerical range. The scores of different facial components are transformed into a common domain with tanh normalization. The fusion of most discriminative component rather than the fusion of all facial components is selected.

\section{Results and Discussions}

A database is created for storing the composite sketch and face photos. The component based approach check the computer generated sketch matches with the face photos. First load the corresponding image, if it is a color image convert into greyscale image. The time required for processing greyscale image is less when compared to color image. The sketch and photos can be normalized using transformation like rotation and scaling to handle variations in scale and pose. The sketch constructed by different user is different. The quality of sketch not only depends on the software kit but also the background of the user. The sketch constructed by asian user is different from a non asian user. An active shape model is used to de-tect landmarks. In component localization face is decomposed into four components hairsytle, eyes, nose and mouth. Component feature extraction divide the face area into small regions from which histogram is extracted and concatenated into single. LBP having some limitations features computed using single scale may not able to detect the dominant texture features. Features extracted from single channel capture only the monochromatic intensity information which limit the recognition performance. The multi-resolution representation based LBP can be obtained by varying the sample radius, $\mathrm{R}$, and combining the LBP image. Multiscale LBP is more accurate than single LBP. The multiscale LBP representation method can be realised in two ways. First, it can be accomplished by increasing the radius of the operator. Alternatively one can down-sample the original image with interpolation and then apply an LBP operator of fixed radius.

\subsection{Discussions}

While examining the discriminative ability of facial components the proposed approach gives more accuracy. Among the facial components,nose, hair, mouth having high discriminability than shape and eye. Hair is not widely used due to over time lapse in photo to photo matching. The proposed system uses the hair component because sometimes it will be heplfull for determining the identity of suspect. The facial component eye is more complicated than other components. Nose is the most stable component. The proposed approach shows eye component is having more difficulty in choosing than other facial component.

An active shape model is used to detect facial landmarks. These landmarks form a distinct shape. Feature extraction of each facial component is obtained using a multiscale binary operator. MLBP help to capture all spatial information of each facial component. For each image calculate the euclidean distance between the test image and image in the database. Check if interpolation is needed, then calculate the 
interpolation weights and interpolation pixel values. The PNN is a feed forward network consists of input layer, hidden layer and output layer. The hidden layer calculates the kernel value. The matching score of each facial component is calculated based on normalized histogram.

Table 4.1 System Comparsion

\begin{tabular}{|l|l|l|l|}
\hline & Hand Drawn Sketches & Forensic Sketches & Proposed Method \\
\hline Composite database & No & No & Yes \\
\hline Face normalization & No & No & Yes \\
\hline Component localization & Manual & Manual & Automatic \\
\hline Performance rate & Low & Low & High \\
\hline
\end{tabular}

The Table shows the comparison between previous work and proposed method. Artist can draw the sketch with exact shape. Hand drawn sketches lack detailed texture information especially around forehead and cheeks. During the drawing of forensic sketch,the witness doesn't recall the exact apperance of the criminal. This will lead mismatch for the depiction and understanding of facial components. The proposed component based representation uses normalization, component localization, per component feature extraction and similarity calculation. Normalization is performed for handling variations in pose and scale. The component localization steps performs face decomposition. A block based feature extraction is performed for each image. The component similarity is calculated for each facial component. The proposed method uses intensity information for handling the changes in facial appearance. A supervised algorithm for matching the sketch to photos gives more accurate performance.

\section{Conclusion}

Automated face recognition technique is widely used in law enforcement agency. A component based approach for matching sketches to face photos using PNN classifier is addresssed. Facial landmarks are detected using ASM. Features of each facial components are extracted using multiscale local binary pattern. MLBP help to and local texture information of each facial component. Component similarities of each facial component is calculated and similarity score of each component can be obtained. PNN classier based on radial basis network help us to reduce the training time. In order to train and test the network, a correlation based template matching is conducted using edge gradient representation of the image. The PNN learns from the training data instantaneously. This speed of learning gives the PNN the capability of adapting its learning in real time, deleting or adding training data as new conditions arises.

In future, the work can be extended by choosing a different classifier.The proposed work can be extended by adding other informations of the suspect like age.race etc. Another enhancement is to study the aging problem of suspects,

\section{References}

[1]. D. Mcquiston, L. Topp, and R. Malpass, "Use of facial composite systemsin US law enforcement agencies,” Psychology, Crime and Law,vol. 12, no. 5, pp. 505-517, 2006.

[2] A. K. Jain, B.Klare, andU. Park, "Face matching and retrieval in forensicsapplications," IEEE Multimedia, vol. 19, no. 1, p. 20, Jan. 2012.

[3] B. Klare, Z. Li, and A. Jain, "Matching forensic sketches to mug shotphotos," IEEE Trans. Pattern Anal. Mach. Intell., vol. 33, no. 3, pp.639-646, Mar. 2011

[4] H. A. Rowley,"Neural Network Based Face Detection, Neural network BasedFace Detection", School of Computer Science, Computer Science Department, Carnegie Mellon University, Pittsburgh, Pa, USA, 1999.

[5] Topi Maenpaa and Matti Pietikainen." Multi-scale binary patterns for texture analysis". In SCIA, pages , 2003. 